\title{
ON SEPARABLE NONCYCLIC EXTENSIONS OF RINGS
}

\author{
GEORGE SZETO and YUEN-FAT WONG
}

(Received 17 January 1982; revised 23 July 1982)

Communicated by R. Lidl

\begin{abstract}
The separable cyclic extension of rings is generalized to a separable noncyclic extension of rings: a crossed product with a factor set over a ring (not necessarily commutative). A representation of separable idempotents for a separable crossed product is obtained, and simplifications for some special factor sets are also given.
\end{abstract}

1980 Mathematics subject classification (Amer. Math. Soc.): 16 A 16.

\section{Introduction}

Let $R$ be a ring with 1 (not necessarily commutative), $\rho$ an automorphism of order $n$ of $R$ for some integer $n$. The separability of the cyclic extension $R[i, \rho]$, has been intensively investigated (Parimula and Sridharan (1977), Nagahara and Kishimoto (1978), Szeto (1980), Szeto and Wong (1982)), where $r i=i(r) \rho$ for each $r$ in $R,\left\{1, i, i^{2}, \ldots, i^{n-1}\right\}$ is a free basis of $R[i, \rho]$ over $R, i^{n}=b$ which is a unit in the center $C$ of $R$ and $(b) \rho=b$. The purpose of the present paper is to continue the above investigation to a noncyclic extension: a crossed product $\Delta(R, G)$, where $G$ is a finite automorphism group (not necessarily cyclic) with factor set $f: G \times G \rightarrow U(C)$, the set of units of the center $C$ of $R$. Our study includes cyclic extensions, crossed products over a commutative ring (DeMeyer and Ingraham (1971), Chapter 3), and crossed products with trivial factor set (Kanzaki (1964), Section 3).

(C) Copyright Australian Mathematical Society 1983 


\section{Preliminaries}

Let $R$ be a ring with $1, C$ the center of $R, G\left(=\left\{g_{1}, g_{2}, \ldots, g_{n}: g_{1}=\right.\right.$ the identity of $G$ for some integer $n\}$ ) an automorphism group of $R$, and $R^{G}=\{r$ in $R$ such that $(r) g_{i}=r$ for each $g_{i}$ in $\left.G\right\}$. A crossed product $\Delta(R, G)$ with factor set $f: G \times G \rightarrow U(C)$, the set of units of $C$, is a free ring with a basis $\left\{U_{i}\right.$ : $i=1, \ldots, n\}$ over $R$ such that $r U_{i}=U_{i}\left((r) g_{i}\right)$ for each $r$ in $R$, and $U_{i} U_{j}=$ $U_{k} f\left(g_{i}, g_{j}\right)$, where $g_{i} g_{j}=g_{k}$ and $f\left(g_{i} g_{j}, g_{m}\right)\left(f\left(g_{i}, g_{j}\right)\right) g_{m}=f\left(g_{i}, g_{j} g_{m}\right) f\left(g_{j}, g_{m}\right)$ for all $g_{i}, g_{j}, g_{m}$ in $G$. We note that $\Delta(R, G)$ is associative if and only if the above equation holds. Let $S$ be a subring with 1 of $R$. Then $R$ is called a separable extension of $S$ if there exist elements $\left\{a_{i}, b_{i}\right.$ in $R$ such that $i=1, \ldots, m$ for some integer $m\}$, such that $t\left(\sum a_{i} \otimes b_{i}\right)=\left(\sum a_{i} \otimes b_{i}\right) t$ for each $t$ in $R$ and $\sum a_{i} b_{i}=1$ where $\otimes$ is over $S$ (Szeto and Wong (1982)). Such an element $\Sigma a_{i} \otimes b_{i}$ is called a separable idempotent for $R$ over $S$. A ring $R$ with 1 and with a finite automorphism group $G$ is called a Galois extension over $R^{G}$ if there exist elements $\left\{a_{i}, b_{i}\right.$ in $R: i=1, \ldots, m$ for some integer $m\}$ such that $\sum a_{i} b_{i}=1$ and $\sum a_{i}\left(\left(b_{i}\right) g_{j}\right)=0$ whenever $g_{j} \neq g_{1}$ (DeMeyer (1965), (1966)). Since $(C) g_{i}=C$ for each $i, G$ induces an automorphism group of $C$. The Kanzaki hypothesis (Kanzaki (1964), page 110) on $R$ means that $R$ is an Azumaya $C$-algebra (central separable) and $C$ is Galois over $C^{G}$ with Galois group induced by and isomorphic with $G$. Throughout, we assume that $R$ is a ring with 1 and $G$ an automorphism group of $R$.

\section{Separability of crossed products}

Under the Kanzaki hypothesis on $R$, we shall show a necessary and sufficient condition for $\Delta(R, G)$ being a separable extension over $R$. It is easy to see that $\Delta(R, G)$ has an identity $U_{1} a^{-1}$ so that $R$ is embedded in $\Delta(R, G)$, where $a=f\left(g_{1}, g_{1}\right)$. We begin with a representation of a separable idempotent for a separable crossed product $\Delta(R, G)$ over $R$.

THEOREM 1. Under the Kanzaki hypothesis on $R$, the element $x\left(=\Sigma U_{i} \otimes U_{j} b_{i j}\right.$ : $i, j=1, \ldots, n$ and $b_{i j}$ are in $\left.R\right)$ is a separable idempotent for $\Delta(R, G)$ if and only if

(1) $b_{i j}=0$ whenever $g_{j} \neq g_{i}^{-1}$, and $b_{i j}$ are in $C$,

(2) $b_{11^{\prime}}=\left(\left(f\left(g_{k}, g_{1}\right)\right)^{-1} g_{1}^{-1}\right) f\left(g_{i}^{-1}, g_{k}\right)\left(b_{i i^{\prime}} g_{k}\right)$, where $g_{1^{\prime}}=g_{1}^{-1}, g_{i^{\prime}}=g_{i}^{-1}$, and $g_{i}=g_{k} g_{1}$, and

(3) $a \cdot \Sigma_{1} f\left(g_{1}, g_{1}^{-1}\right)\left(\left(f\left(g_{k}, g_{1}\right)\right)^{-1} g_{1}^{-1}\right) f\left(g_{i}^{-1}, g_{k}\right)\left(b_{i i}, g_{k}\right)=1$, where $a=$ $f\left(g_{1}, g_{1}\right)$ and $g_{i}=g_{k} g_{1}$. 
Proof. Let $x$ be a separable idempotent for $\Delta(R, G)$ over $R$. Since $b x=x b$ for each $b$ in $R, \Sigma_{i, j} b\left(U_{i} \otimes b_{j}\right) b_{i j}=\Sigma_{i, j}\left(U_{i} \otimes U_{j}\right) b_{i j} b$. Hence $\Sigma_{i, j}\left(U_{i} \otimes U_{j}\right)\left(b g_{i} g_{j}\right) b_{i j}$ $=\Sigma_{i, j}\left(U_{i} \otimes U_{j}\right) b_{i j} b$. In particular, taking $b$ in $C$, we have that $\left(b g_{i} g_{j}\right) b_{i j}=b_{i j} b$, so $b_{i j}\left(b-\left(b g_{i} g_{j}\right)\right)=0$. Hence $b_{i j}$ is in the annihilator ideal $I$ of the ideal $J$ generated by $\left\{b-\left(b g_{i} g_{j}\right): b\right.$ in $\left.C\right\}$. By hypothesis, $R$ is Azumaya over $C$, so $I=I_{0} R$ (DeMeyer and Ingraham (1971), Corollary 3.7, page 54) where $I_{0}=I \cap$ $C$. Noting that $I_{0}$ is the annihilator ideal of $J$ in $C$, we have that $I_{0}=\{0\}$ (DeMeyer and Ingraham (1971), Proposition 1.2, page 81) because $C$ is Galois over $C^{G}$ with Galois group induced by and isomorphic with $G$. This implies that $b_{i j}=0$ whenever $g_{j} \neq g_{i}^{-1}$. Let $i^{\prime}=j$ in case $g_{j}=g_{i}^{-1}$. Then $x=\Sigma_{i}\left(U_{i} \otimes U_{i^{\prime}}\right) b_{i i^{\prime}}$, Thus we can write $b_{i}$ for $b_{i i^{\prime}}$ so that $x=\Sigma_{i}\left(U_{i} \otimes U_{i}\right) b_{i}$. Again, from the equation $b x=x b$ for each $b$ in $R, b_{i}$ are in $C$. Moreover, for each $U_{k}, U_{k} x=x U_{k}$, so $\Sigma_{1}\left(U_{k} U_{1} \otimes U_{1^{\prime}}\right) b_{1}=\Sigma_{i} U_{i} \otimes U_{i^{\prime}} U_{k}\left(b_{i} g_{k}\right)$. Let $g_{i}^{-1} g_{k}=g_{j}$. Then $g_{i}=g_{k} g_{j}^{-1}$. Thus $U_{i^{\prime}} U_{k}=U_{j} f\left(g_{i}^{-1}, g_{k}\right)$ and $U_{i}=U_{k} U_{j^{\prime}}\left(f\left(g_{k}, g_{j}^{-1}\right)\right)^{-1}$. This implies that

$$
\begin{aligned}
\sum_{1}\left(U_{k} U_{1} \otimes U_{1^{\prime}}\right) b_{1} & =\sum_{i} U_{k} U_{j^{\prime}}\left(f\left(g_{k}, g_{j}^{-1}\right)\right)^{-1} \otimes U_{j}\left(g_{i}^{-1}, g_{k}\right)\left(b_{i} g_{k}\right) \\
& =\sum_{i}\left(U_{k} U_{j^{\prime}} \otimes U_{j}\right)\left(f\left(g_{k}, g_{j}^{-1}\right)\right)^{-1} g_{j} f\left(g_{i}^{-1}, g_{k}\right)\left(b_{i} g_{k}\right) .
\end{aligned}
$$

Let $U_{j}=U_{1^{\prime}}$. Then, $g_{j}=g_{1}^{-1}, g_{1}=g_{j}^{-1}, U_{1}=U_{j^{\prime}}$ and $U_{1^{\prime}}=U_{j}$. Hence $U_{k} U_{1} \otimes U_{1^{\prime}}$ $=U_{k} U_{j^{\prime}} \otimes U_{j}$. Thus $b_{1}=\left(f\left(g_{k}, g_{1}\right)\right)^{-1} g_{1}^{-1} f\left(g_{i}^{-1}, g_{k}\right)\left(b_{i} g_{k}\right)$ for each 1 , where $g_{i}=$ $g_{k} g_{1}$. Furthermore, noting that $\Sigma_{1} U_{1} f\left(g_{1}, g_{1}\right) b_{1}=U_{1} a^{-1}$, we have that $a \cdot \Sigma_{1} f\left(g_{1}, g_{1}^{-1}\right)\left(f\left(g_{k}, g_{1}\right)\right)^{-1} g_{1}^{-1} f\left(g_{i}^{-1}, g_{k}\right)\left(b_{i} g_{k}\right)=1$. This proves the necessity. The sufficiency is immediate by reversing the above arguments.

From Theorem 1, the coefficients of $x$ are in $C$ and the factor set $f: G \times G \rightarrow$ $U(C)$, so $\Delta(R, G)$ is separable over $R$ if and only if $\Delta(C, G)$ is separable over $C$. Next, we study the separability of $\Delta(R, G)$ for some types of factor sets $f$. A factor set $f$ is called I-symmetric if $f\left(g_{i}^{-1}, g_{j}\right)=f\left(g_{j}^{-1}, g_{i}\right)$ for all $g_{i}, g_{j}$ in $G$. ( $f$ can be considered as a function on entries of a matrix with row index $\left\{1^{\prime}, \ldots, n^{\prime}\right\}$ and column index $\{1, \ldots, n\}$ where $g_{i^{\prime}}=g_{i}^{-1}$.) A factor set $f$ is called a scalar factor set if $f\left(g_{i^{\prime}}, g_{l}\right)$ is a constant for $i=1, \ldots, n$. The following property of $f$ is easy to verify:

Lemma 2. Let $f$ be a factor set such that $f\left(g_{1}, g_{1}\right)=a$. Then $\left(a f\left(g_{i}, g_{i^{\prime}}\right)\right) g_{i}=$ af $\left(g_{i^{\prime}}, g_{i}\right)$ for each $i$. 
THEOREM 3. Assume that $f: G \times G \rightarrow U\left(C^{G}\right)$ such that $f$ is I-symmetric and scalar. If $\Delta(R, G)$ is separable over $R$, then any separable idempotent $x\left(=\Sigma_{j}\left(U_{j} \otimes\right.\right.$ $\left.\left.U_{j^{\prime}}\right) b_{j}\right)$ satisfies

(1) $b_{j}=\left(b_{1}\right) g_{j}^{-1}$ for some $b_{1}$ in $C$ and for each $j$, and

(2) $\sum_{j}\left(b_{1}\right) g_{j}^{-1}=a^{-2}$ where $a=f\left(g_{1}, g_{1}\right)$.

Proof. Since $f(G \times G) \subset U\left(C^{G}\right), a f\left(g_{j}, g_{j}^{-1}\right)=a f\left(g_{j}^{-1}, g_{j}\right)$ for each $j$ by the lemma. Hence $f\left(g_{j}, g_{j}^{-1}\right)=f\left(g_{j}^{-1}, g_{j}\right)=a$ (for $f$ is scalar). Since $f\left(g_{i}^{-1}, g_{k}\right) f\left(g_{j}^{-1}, g_{k}^{-1}\right)=f\left(g_{j}^{-1} g_{k}^{-1}, g_{k}\right) f\left(g_{j}^{-1}, g_{k}^{-1}\right)=f\left(g_{j}^{-1}, g_{k}^{-1} g_{k}\right) f\left(g_{k}^{-1}, g_{k}\right)=$ $a^{2}, f\left(g_{i}^{-1}, g_{k}\right)=a^{2}\left(f\left(g_{j}^{-1}, g_{k}^{-1}\right)\right)^{-1}$. But $f$ is $I$-symmetric, so $f\left(g_{j}^{-1}, g_{k}^{-1}\right)=$ $f\left(g_{k}, g_{j}\right)$. Then, conditions (2) and (3) in Theorem l imply that $b_{j}=$ $\left(f\left(g_{k}, g_{j}\right)\right)^{-2} a^{2}\left(b_{i} g_{k}\right)$ and $1=a^{4} \Sigma_{j}\left(f\left(g_{k}, g_{j}\right)\right)^{-2}\left(b_{i} g_{k}\right)$ where $g_{i}=g_{k} g_{j}$. Thus $1=a^{2} \Sigma_{j} b_{j}$, and so $\Sigma_{j} b_{j}=a^{-2}$. Taking $i=1$, we have that $g_{k}=g_{j}^{-1}$. Hence, $1=a^{4} a^{-2} \Sigma_{j}\left(b_{1} g_{j}^{-1}\right)=a^{2} \Sigma_{j}\left(b_{1} g_{j}^{-1}\right)$, so $\sum_{j}\left(b_{1} g_{j}^{-1}\right)=a^{-2}$. Also, $b_{j}=$ $a^{-2} a^{2}\left(b_{1} g_{j}^{-1}\right)=b_{1} g_{j}^{-1}$.

Condition (1) means that each coefficient $b_{j}$ of $x$ is determined by $b_{1}$, and condition (2) implies that the trace of $b_{1}$ is $a^{-2}$. It can be verified that the converse of Theorem 3 holds for any constant factor set $f$.

THEOREM 4. If $f: G \times G \rightarrow U\left(C^{G}\right)$ is a constant, then the converse of Theorem 3 holds.

Assume $n c=1$ for some $c$ in $C$. Then the trace of $c a^{-2}$ is $a^{-2}$. Thus $\Delta(R, G)$ is a separable extension over $R$ by Theorem 4 . We conclude the paper with an example to demonstrate our results. Let $R[i, \rho]$ be a generalized quaternion algebra (Parimula and Sridharan (1977), Szeto (1980)), where $\{1, i\}$ is a basis for $R[i, \rho]$ over $R, \rho$ an automorphism of $R$ of order $2, r i=i(r \rho)$ for each $r$ in $R$ and $i^{2}=b$ in $U\left(C^{\rho}\right)$. We define $f:\langle\rho\rangle \times\langle\rho\rangle \rightarrow U(C)$ by $f\left(\rho^{0}, \rho\right)=f\left(\rho, \rho^{0}\right)=$ $f\left(\rho^{0}, \rho^{0}\right)=1$ and $f(\rho, \rho)=b$. Then it is easy to see that $f$ is a factor set for the crossed product $\Delta(R,\langle\rho\rangle)$ with basis $U_{0}=U_{\rho^{0}}, U_{1}=U_{\rho}$ such that the identity is $U_{0}$ and that $R[i, \rho]$ is isomorphic with $\Delta(R,\langle\rho\rangle)$ with factor set $f$ under $\alpha$ : $R[i, \rho] \rightarrow \Delta(R,\langle\rho\rangle)$ where $\alpha(x+i y)=U_{0} x+U_{1} y$ for $x$ and $y$ in $R$.

\section{References}

F. R. DeMeyer (1965), 'Some notes on the general Galois theory of rings,' Osaka J. Math. 2, 117-127.

F. R. DeMeyer (1966), 'Galois theory in separable algebras over commutative rings,' Illinois J. Math.

2, 287-295. 
F. R. DeMeyer and E. Ingraham (1971), Separable algebras over commutative rings (Lecture Notes in Mathematics 181, Springer-Verlag, Berlin-Heidelberg-New York).

T. Kanzaki (1964), 'On commutor rings and Galois theory of separable algebras,' Osaka J. Math. 1, 103-115.

T. Nagahara and K. Kishimoto (1978), 'On free cyclic extensions of rings,' Math. J. Okayama Univ., 1-25.

S. Parimula and R. Sridharan (1977), 'Projective modules over quaternion algebras,' J. Pure Appl. Algebra 9, 181-193.

G. Szeto (1980), 'A characterization of a cyclic Galois extension of commutative rings,' J. Pure Appl. Algebra 16, 315-322.

G. Szeto and Y. F. Wong (1982), 'On separable cyclic extensions of rings,' J. Austral. Math. Soc. Ser. A 32, 165-170.

Department of Mathematics

Bradley University

Peoria, Illinois 61625

U.S.A.
Department of Mathematics

DePaul University

Chicago, Illinois 60637

U.S.A. 